\title{
ETUDE DÉTAILLÉE DU MOUVEMENT ET DE LA STABILISATION DU FRONT DE PRÉCIPITATION LORSQUE DEUX RÉACTIFS DIFFUSENT L'UN VERS L'AUTRE DANS UN GEL
}

\author{
par J. SALVINIEN et J.J. MOREAU \\ [Facullé des Sciences de Montpellier]
}

\section{S()MMIAIRL}

\begin{abstract}
Juscu’ici, le problème de diffusion double avec précipilation n’a reçu (jue des solutions particlles. La présente étude a pour but d'en (lomer une solution beaucoup) plus complète.
\end{abstract}

\section{Introduction.}

L'importance accruc des méthodes d'analyse immunochimicfue par diffusion pure ou accompagnéc d'électrophorise, l'extension possible de ces méthodes à d'autres domaines de la chimie, l'intérêt de l'élude des précipitations avec ou en l'alsence de gel, invitent à ajperofondir plusicurs problèmes fondamentaux de diffusion avec précijpilation.

Nous laisserons de côté le problème dit de di/fusion simple car, en l'absence de complications telles que la formation de précipitations périodicpues, on peut le considérer comme très approximativement résolu $\left({ }^{1}\right)\left({ }^{2}\right)\left({ }^{*}\right)$.

Par contre, le probleme de difrusion double n'a reçu jusqu'ici que des solutions particlles sur lesquelles nous reviendrons brievement. Nous donnerons ensuite des solutions malliématiques jolus détaillées qui seront confrontées avec les faits.

l)ans ce (pui suil, nous supp)oserons que les conditions suivantes sont toujours réalisćes:

Au temps $l=0$, les réactifs $I R$ el $I^{\prime}$ partent respeclivement de deux réservoirs limilés, vers le champ de diflusion, par deux surfaces planes et parallèles $A$ el $\Lambda^{\prime}$ (lig. 1). Ils progressent l'un vers l'autre dans un licjuide ou un gel homogines, défourvus de réactif interne. Ides concentrations $C_{0}$ el $\mathrm{C}_{0}{ }^{\prime}$ de $R$ el $\mathrm{I}^{\prime}$ sur les hases de dejarl $\mathrm{A}$ el $\mathrm{A}^{\prime}$ sont. maintenues constantes pendant Loule la durée de l'expérience.

(*) Une solution encore plus salisfaisante de ec problime est domnéc à ce colloque par Rosa $\Lambda$ Uiustin et Sirens.
Lors de la rencontre des réactifs, la réaction est immédiate el complète. Le précipité est considéré comme tolalement insoluble, sauf dans la phase initiale de toute premiere précipitation oì l'on peut éventuellement laire intervenir un produit de solubilité $s$ très petil, car les flux opposés des réactifs qui se rencontrent sont alors eux-mèmes très laibles. Par la suite, ces flux deviennent beaucoup) j) lus grands el $s$ jeut être négligé.

Dans ces conditions, lorsque le front de précipiLation IF s'est formé, il sépare le champ) de diflusion en deux domaines. Le domaine situé entre $\Lambda$ et $I *$, renferme $R$ à l'exclusion de $R^{\prime}$. Le domaine situc entre $\Lambda^{\prime}$ et $F$, renferme $R^{\prime}$ à l'exclusion de $R$. Sur le front fi lui-meme, les concentrations de $R$ et de $R^{\prime}$ sont mulles. Nous désignerons par (: la concentration variable de $\mathrm{R}$ dans le premier domaine. et par $C^{\prime}$ celle de $R^{\prime}$ dans le second domaine. Les surfaces d'épuiconcentrations sont évidemment des plans parallides à $\Lambda$ el $A^{\prime}$. Il s'agil donc de diffusions planes unidimensionnelles suivant la clirection $A \Lambda^{\prime}$.

Nous applipuerons l'épuation différenticlle de Fick, ce qui revient à admettre que les coefricients de dilfusion I) el $\mathrm{D}^{\prime}$ des réactil's sont constants. Cetle hypothèse ne peut être rigoureusement exacte car $\mathrm{D}$ et I)' sont des fonctions lentement variables de $C$ et de C'. En outre, l'un des réactif's est souvent astreint à traverser une zone de précipité dans lapuelle son coeflicient de diftusion est un peu modifié. Mais nous ne pouvons obtenir une rejoésentation quantilative parfaite d'un phénomene aussi complexe que celui (fui nous intéresse el les variations précédentes de I) et I)' sont le plus souvent négligeables devant d'autres causes possibles d'erreurs.

Lorsque R el $R^{\prime}$ se rencontrent, il apparait une zone plane $F_{1}$, plus ou moins étroite, de premiere précipilalion. Pous, après une période d'organisalion, ou voit se deplacer un front li (pui, en general, linit par se lixer sur une position stationnaire linile 
$F_{p}$ correspondant à un double régime permanent de diffusion des deux réactil's. Nous appelons fronl de régime permanenl celle posilion linte $F_{p}$, de $F$. $\Lambda_{u}$ cours de prérédentes études nous avions déterminé les positions de $F_{1}$ et de $F$, mais nous n'avions pas recherché less conditions générales de stabilité du front, ni les lois de son évolution entre les positions $F_{1}$ et $F_{p}$. Lo présent mémoire a précisément pour but de combler ees lacunes.

Dans les paragraphess suivants, nous exprimerons les concentrations en normalités.

\section{Front de régime permanent.}

Nous avons indiqué une méthorle générale permettant de determiner les fronts de regime permanent pour des systimes de diflérentes configurations $\left(^{2}\right)$. Dans le cas particulierement simple de la double diffusion iniolimensionnelle, la position de $F_{p}$, est fixés par la relation:

$$
\frac{\mathrm{F}_{p} \mathrm{\Lambda}}{\mathrm{I}_{p}{ }_{p}^{\prime} \mathbf{\Lambda}^{\prime}}=\frac{\mathrm{D}}{\mathrm{D}^{\prime}} \frac{\mathrm{C}_{w}}{\mathrm{C}^{\prime}{ }_{o}}
$$

\section{Zone de première précipitation}

Pour la déterminer, il est nécessaire de laire appel à une hypothèse physique relative à la solubilité tris faible du précipité.

S'il s'agit d'un précipité minéral, il est naturel d'employer la notion de produit de solubilité. Cetle notion est étendue un peu arbitmairement au cas où le précipité se forme dans un gel. ()n sait que le seuil de précipitation est alors souvent plus élevé que dans l'eau. Mais on peut admettre que, si le rapport $C / /\left(C^{\prime}\right.$ ne varie pas trop), le produit c.C' est sensiblement constant an seuil de précipitation. Cette hypothese revient à introduire un produit apparenl de solubilité s, généralement plus élevé que le vérilable produit de solubilité s mesuré en l'absence de gel.

Or, on peut montrer que, juste avant le début de la précipitation, aux points du gel oì les deux réactifs viennent de s’interpénétrer légèrement, le produit très petit $C^{\prime \prime} C^{\prime}$ présente un maximum $\mathrm{M}$ dans un plan $F_{M}$ parallele à $\Lambda$ et $\Lambda^{\prime}$. La position de $\mathrm{F}_{\mathrm{M}}$ est indépendante du temps $t$ et des concentrations à l'origine $C_{0}$ et $C_{0}{ }^{\prime}$. C'est dans ce plan que doit normalement s'amorcer la précipitalion. $\mathrm{F}_{\mathrm{M}}$ se confond done avec le front théorique $F_{1}$ de premiere précipitation. Le calcul $\left({ }^{3}\right)$ conduit à la relation très simple:

$$
\frac{F_{1} A}{F_{1} A^{\prime}}=\frac{D}{D^{\prime}}
$$

Il faut bien préciser que cette relation n'est valable que si l'on peut réellement faire appel à un produit apparent de solubilité.

Le maximum M est peu accusé. Il est d'autant plus applati que les bases de départ $\Lambda$ el $\Lambda^{\prime}$ sont plus aboignées et que les concentrations à l'origine Co et ( ‘ 'sont plus laibles. Il en résulteque l'on observe la lormation d'une zone de première précipitation dont l'épaisseur $\varepsilon$ est une fonction croissante de la distance $\Lambda \Lambda^{\prime}$ et une fonction décroissante de $C_{0}$ et $\mathrm{C}_{0}{ }^{\prime}$. Par excmple, avec le système " $\mathrm{SO}_{4} \mathrm{C}$ (u, ( $\mathrm{Cl}_{2} \mathrm{Ba}$, sel de gélatine à $10 \%$ ", pour $\left(C_{0}=C_{0}{ }^{\prime}=N / 10\right.$ el $\Lambda \Lambda^{\prime}=4 \mathrm{~cm}$, on a oblemu $\varepsilon=0,0993 \mathrm{~cm}$.

Dans beaucoup de cas, la position expérimentale de $F_{1}$, milieu de la zone de première précipitation, coüncide sensiblement avec la position théricyue prévue par l'é(qualion (2). P’our un système donné, la coüncidence parait d'autant meilleure que les réservoirs sont plus rapprochés et les concentrations $C_{0}$ et $C_{0}^{\prime}$ plus élevées.

Cependant, on constale partois de très nets écarts (qui ne paraissent pas attribuables à l'absence d'un produit apparent de solubilité.

$F_{1}$ doit ètre fort sensible à tout engagement chimique prélérentiel du gel sur l'un des réactil's. Par exemple, cill a un coellicient de diffusion presque deux lois plus grand que celui de $\mathrm{NO}_{3} \Lambda \mathrm{g}$. ()r, avec ce systime, on observe un front de premiereprécipitation netlement plus proche du réservoir à cill que du réservoir à $\mathrm{N}()_{3}$ Ag. L'écart entre F, théorique et $F_{1}$ expérimental est considémale. Nous l'attribuons à l'action bien connue de C.IH sur la gélatine. A ce propos, nous jugeons tres intéressante une étude comparative des positions de F pour des systèmes ne difrérent que par la nature du milieu dans lequel s'effectue la diffusion: eau pure, gels de gélatine, de gílose, etc... I a mesure des écarts doit permettre une étude quantilative des réactions entre les réactifs et le colloüde formant le gel.

Si, au lieu d'éludier la diffusion d'électrolytes ordinaires, on étudie celle des réactilss de l'immunochimie, la notion de produit de solubilité ne peut phus ètre conservée. La précipitation doit s'amoreer en des points oì les concentrations $C$ et et $C^{\prime}$, exprimées en é(puivalents-gramme, sont égales (point d'équivalence). Il est lacile de montrer que, dans ce cas, la position du front $F_{1}$ est déterminée par la relation

$$
\frac{F_{1} A}{F_{1} A^{\prime}}=\frac{\sqrt{D}}{\sqrt{D^{\prime}}}
$$

\section{Étude de la stabilité du front.}

L'un de nous $\left({ }^{3}\right)$ avait lait remarquer que si les concentrations à l'origine $C_{0}$ et $C_{0}^{\prime}$ sont égales, les fronts $F_{p}$ et $F_{1}$ coîncident. (formules 1 el 2 ) Par 
conséefuent, le lront doit s'épaissir sur place, sans se déplacer. Cetle prévision paraì souvent vériliée par l'expérience quand les réservoirs sont peu éloignés el les coeflicients de diffusion pas trop différents l'un de l'autre. Mais, dans le cas contraire, on peut observer une sorte de va et vient du front. C'est une des raisons pour lespuelles il nous a paru nécessaire de faire une élude serrée des conditions de stabilité du front.

Posons $\Lambda A^{\prime}=a$.

Pour un point M quelcongue de la droile $\Lambda A^{\prime}$ (ligs. 1) nous poserons :

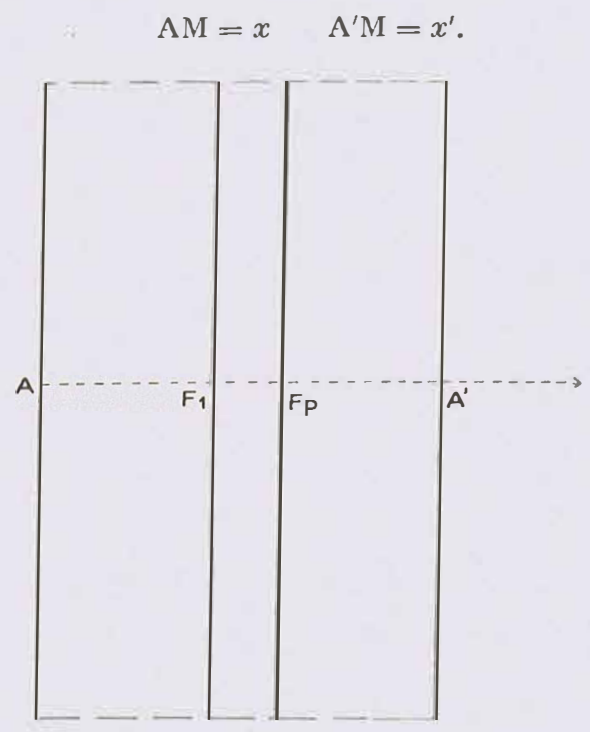

Fig. 1.

On a évidemment:

$$
x+x^{\prime}=a .
$$

$\Lambda$ sauche du front $\mathrm{F}$, la concentration $\mathrm{C}(x, l)$ du réactif R vérilie l'écfualion de Fick:

$$
\text { D) }{ }^{3} \mathrm{C}^{2} \cdot \mathrm{x}^{2}=\frac{\mathrm{iC}}{11 /}
$$

dans la région $F \Lambda^{\prime}$, la concentration $C^{\prime}\left(x^{\prime}, l\right)$ du réaclif $\Omega^{\prime}$ vérilie la relalion similaire:

$$
\mathrm{D}^{\prime} \frac{\partial^{2} \mathrm{C}^{\prime}}{\partial x^{\prime 2}}=\frac{\partial \mathrm{C}^{\prime}}{\partial t}
$$

Sur le front F lui-même, nous écrivons l'égalilé des llux et la mullité des concentrations:

$$
\text { I) } \frac{\partial C_{1}}{\partial x}=D^{\prime} \frac{\partial C^{\prime}}{\partial x^{\prime}} \quad \text { el } \quad \text { (i) } \quad \mathrm{C}=\mathrm{C}^{\prime}=\mathbf{0} \text {. }
$$

Les conditions aux limiles en $\Lambda$ et $A^{\prime}$ sont:

$$
\mathrm{C}(0, \iota)=\mathrm{C}_{w} \quad \text { el }
$$

Pour résoudre le probleme, nous nous reporlons a la solution réduile du probleme du mur. Pour cela, nous considérons la fonclion $\gamma(\zeta, \quad 0)$ salislaisant à

$$
\frac{\partial^{2} \gamma}{\partial \xi^{2}}=\frac{\partial \gamma}{\partial 0} \quad \text { pour } \quad 0 \leqslant \xi \leqslant 1
$$

avec $\gamma(\stackrel{r}{\xi}, 0)=0$ quelque soilţ.

$$
\gamma(0,0)=1 \quad \text { el } \quad \gamma(1,0)=0 \text {, quelque soil } 0 .
$$

y est une fonclion numéricpue bien délinie dont on peul construire divers développements ulilisables pour le calcul.

Recherchons alors une solution à fromt lixe. Posons $A F=l, A^{\prime} F=l^{\prime}$, avec $/+l^{\prime}=a$.

Si la solution cherchée existe, la concentration $C(x, l)$ sur $\Lambda F$ est solulion a'mu probleme du mur et peul s'exprimer an moyen de la fonclion $\gamma(\xi, \rho)$ délinic ci-dessus.

Posons

$$
\xi=\alpha x, \quad \text { el } \quad 0=\beta \iota \text {. }
$$

La lonclion $C(x, l)=k \gamma(\alpha x, \beta l)$ donne

$$
\frac{\lambda \mathrm{C}}{\lambda x}=k \alpha \frac{i \gamma}{\jmath \xi \xi} \quad \text { puis } \quad \text { (11) } \quad \frac{\partial^{2} \mathrm{C}}{\partial x^{2}}=k \alpha^{2} \frac{\lambda^{2} \gamma}{\partial \xi^{2}}
$$

el

$$
\frac{\partial \mathrm{C}}{\partial t}=k \beta \frac{\partial \gamma}{\partial \theta}
$$

d'oì, d'aprìs (?),

$$
\frac{1}{\alpha^{2}} \frac{\partial^{2} \mathrm{C}}{\partial x^{2}}=\frac{1}{\beta} \frac{\partial \mathrm{C}}{\partial t}
$$

C. virifie donc (4) si $\beta / \alpha^{2}=D$, cl. (7) si $k=C_{0}$. J'écfualion (6) est vériliéce si

$$
\mathrm{C}(/, t)=k \gamma(\alpha /, \rho l)=0
$$

(fuclefue soil $l$, c'est-ì-dlire si $\alpha /=1$.

En réstmé, on doil avoir

$$
k=\mathrm{C}_{\prime \prime} \quad \alpha=\frac{1}{\mathrm{f}} \quad \beta=\mathrm{J} \alpha^{2}=\frac{\mathrm{D}}{f^{2}} .
$$

()n aurail de même, avec le réaclif $R^{\prime}$

$$
k^{\prime}=C^{\prime}{ }_{1} \quad \alpha^{\prime}=\frac{1}{l^{\prime}} \quad \beta^{\prime}=\frac{D^{\prime}}{f^{\prime 2}},
$$

ponr vérilier les condilions (4t) (6) el (8).

Reste à assurer la condilion (5)). ()r, sur li, nous avolls

$$
\begin{aligned}
& \text { D) } \frac{\lambda C}{1, x}=D k \alpha \frac{n \gamma}{n \xi}(1, \beta t)
\end{aligned}
$$

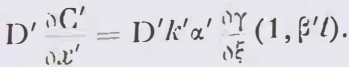

(i)) sera donc verifice quelpue soit / si 


$$
\mathrm{D} k \alpha=D^{\prime} k^{\prime} \alpha^{\prime} \quad \text { et } \beta=\beta^{\prime} .
$$

Récipropuement, loule solution de l'ensemble. des rolalions (11) (15) et (18) fournit une solution à front fixe du probleme initial. si

Les solulions de (14) et (15) vérilieront bien (18)

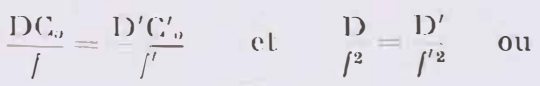

$$
\begin{aligned}
& f^{\prime}=\frac{V D}{V D)^{\prime}}=\frac{D C^{\prime \prime}}{D^{\prime}\left(C_{*}^{\prime \prime}\right.}
\end{aligned}
$$

Il en résulle que la condilion nécessaire of suffisante pour oblenir la lixile du front est:

$$
\mathrm{C}_{0} \sqrt{\mathrm{D}}=\mathrm{C}_{0}^{\prime} \sqrt{\mathrm{D}^{\prime}}
$$

Toutefois, la condilion (2), (pui silne le front de première précipitation par la considéation d'un produit de solubilite, ne permet pas d'espérer la fixilé absolue du front des son apparilion.

Par contre, la condition (3), concernant l'immunochimice, est compalible avec l'analyse précédente.

\section{Déplacement du front}

Dès que less concentralions ont atteint dans le domaine des valeurs sulfisanles pour que le produit de solubilite s soit négligeable devant clles, le phénomene est regi par les éfualions suivantes, admises jusqu'à présent par lous les auleurs ayant étudic des problèmes analogues.

Soil

$$
\overline{\mathrm{AF}}=\mathrm{X}(l)
$$

l'abscisse du lront F a l'instant l. Sur $\Lambda F$, c'est-ii-dire pour $0<x<\mathrm{X}(l)$, la concentralion $(:(x, l)$ véritic pout toul / l'épuation de lisk

$$
\text { 1) } \frac{11^{2} \mathrm{C}}{11, x^{2}}=\frac{11 \mathrm{C}}{1 \mathrm{c}}
$$

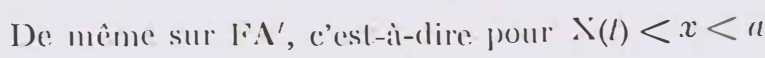

$$
\mathrm{I}^{\prime} \frac{1{ }^{2} \mathrm{C}^{\prime}}{11 \mathrm{x}^{2}}=\frac{\mathrm{i \textrm {C } ^ { \prime }}}{1 \mathrm{il}}
$$

Pour $l=0$, on a la condilion iniliale:

$$
\mathrm{C}(x, 0)=\mathrm{C}^{\prime}(x, 0)=0
$$

Dans les lanches extrêmes $\mathbf{\Lambda}$ el $\mathbf{\Lambda}^{\prime}$ on a les conditions alux limiles

$$
\begin{aligned}
\mathrm{C}((), l) & =\mathrm{C}_{a \prime}, \\
\mathrm{C}^{\prime}(a, t) & =\mathrm{C}_{u}^{\prime}
\end{aligned}
$$

pour tout. $t$.
On a enfin les condilions an fronl de réaction, pour $x=\aleph(l)$ :

$$
\mathrm{C}(\mathrm{X}, t)=\mathrm{C}^{\prime}(\mathrm{X}, t)=0
$$

avece l'igalité dess llux des deux réactifs:

$$
\mathrm{D} \frac{\mathrm{n} C \mathrm{C}}{\mathrm{i} x}(\mathrm{X}, t)=-\mathrm{D}^{\prime} \frac{\mathrm{n} \mathrm{C}^{\prime}}{\mathrm{\lambda} x}(\mathrm{X}, t)
$$

Ce probleme d’integration de deux épuations an derivées partielles differentes (21) el (22) avece des conditions de racordement sur me frontiere inconmue $x=\mathbb{X}(l)$ est a priori d'une grande difricullé. Mentionnons d'abord un cas particulier oi une solulion rigonreuse est possible.

\section{Cas où I) e't I't sonl ćgalltx.}

Si, dans ce cas, on definit une fonclion $\mathrm{K}(x, 1)$ par:

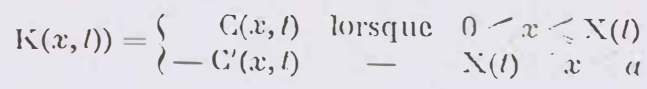

()n voil que celle fonclion verilic pour loul $x$ la meme épualion aux deriveses partielles (21). I,es conditions (26) (2) (27) montrent ru’elle est conlinue sur le fronl F a ansi (pue ses derive parliclles premieres. C̈est done une solulion réguliere de (21) salisfaisant aux conditions intiales cl aux lintles

$$
\begin{array}{lll}
\mathrm{K}(x, 0)=0 & \text { pour } & 0<x<a \\
\mathrm{~K}(0, l)=\mathrm{C}_{a} & \text { pour } & t>0 \\
\mathrm{~K}(a, l)=-\mathrm{C}_{u^{\prime}}{ }^{\prime} & &
\end{array}
$$

Ia recherche de $\mathrm{K}$ constilue done un chassigue problime dil mur.

Nous désignerons par $\gamma(\xi, 0)$ la solulion réduile du probleme: du mur, cesst-it-dire la fonction définie pour

$$
\text { () }-\xi-1, \quad 1=0
$$

et verritiant dans ce domaine l'épualion anx derivées parliclles

$$
\frac{\partial^{2} \gamma}{\partial \xi^{2}}=\frac{\partial \gamma}{\partial 0}
$$

avec les conclitions it la fronliere.

$$
\begin{aligned}
& \gamma(\xi, 0)=0 \\
& \gamma(0,0)=1 \\
& \gamma(1,0)=0
\end{aligned}
$$

On comnaîl pour cetle fonclion les developpoments classicjues:

$$
\gamma(\xi, 0)=1-\xi-\sum_{k=1}^{\infty} \frac{2}{k \pi} e^{-k \varepsilon^{2} 0} \sin k \pi^{\xi} \xi
$$


de calcul numérigue commode si 0 est assez grand et

$$
\gamma(\xi, 0)=\operatorname{crlc} \frac{\xi}{2 \sqrt{0}}+\sum_{n=1}^{\infty}\left(\operatorname{erlc} \frac{2 n+\xi}{2 \sqrt{0}}-\operatorname{erfc} \frac{2 n-\xi}{2 \sqrt{0}}\right)
$$

avec la notation classicpue

$$
\operatorname{erfc} u=1-\operatorname{erf} u=\frac{2}{\sqrt{\pi}} \int_{u}^{\infty} e^{-t z} \mathrm{~d} t
$$

$\Lambda u$ point de vue du calcul numéripue celle dernière série est préférable à la première lorsque o est petit. (On trouve alors facilement la fonction $k$ cherchéc sous la forme

$$
\mathrm{K}(x, \iota)=\mathrm{C}_{v} \gamma\left(\frac{x}{a}, \frac{\mathrm{D} /}{a^{2}}\right)-\mathrm{C}_{u^{\prime}} \gamma\left(\frac{a-x}{a}, \frac{\mathrm{D} /}{a^{2}}\right)
$$

Pour charpue valeur de $\ell$, l'abscisse $X$ du front li soblient comme solution de l'écuation

$$
\mathrm{K}(\mathrm{X}, t)=0
$$

\section{Cas général: $\mathrm{D}$ el $\mathrm{I})^{\prime}$ sont différents.}

Nous devrons nous contenter alors d'une solution approchée (qui fournirail au besoin le premier stacle d'une méthode d'approximations successives), sulfisante pour les besoins présents si le front se déplace assez peu (déplacement total inféricur a $\frac{a}{4}$ pour
lixer les iclées).

Tracons, pour une valeur de $t$, la courbe représentative des variations de $C$ et $C^{\prime}$ en fonction de

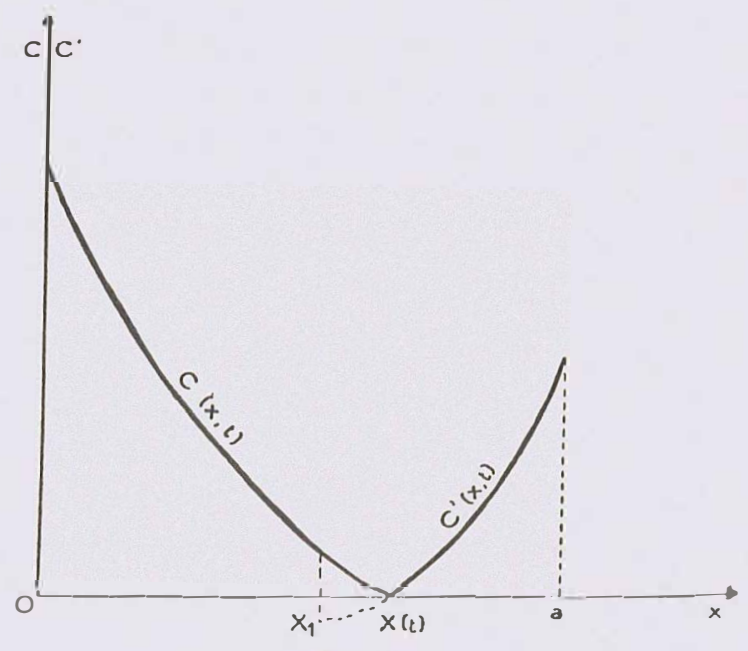

Fig. 2.

$x$ (fig. 2). Si $X(l)$ ne s'éloigne pas $\mid r(p)$ d'une valeur fixe $\mathrm{X}_{1}$, on pourra, entre $\mathrm{X}$ el $\mathrm{X}_{1}$ remplacer les deux courbes par leurs langentes aux points d'abscisse $\mathrm{X}_{1}$ (l'une d'entre elle's est ainsi prolongée en dessous de l'axe des $x$ )

Si on pose

$$
\mathrm{X}(t)-\mathrm{X}_{1}=h(t)
$$

Les conditions (1-(i) sont ainsi remplacées par

$$
\begin{aligned}
& C\left(\mathrm{X}_{1}, l\right)+h \frac{\partial \mathrm{C}}{\partial x}\left(\mathrm{X}_{1}, t\right)=0 \\
& C^{\prime}\left(\mathrm{X}_{1}, l\right)+h \frac{\partial \mathrm{C}^{\prime}}{\partial x}\left(\mathrm{X}_{1}, l\right)=0
\end{aligned}
$$

c'est-à-dire

$$
\frac{C^{\prime}\left(\mathrm{X}_{1}, t\right)}{\frac{\partial \mathrm{C}^{\prime}}{\partial x}\left(\mathrm{X}_{1}, t\right)}=\frac{\mathrm{C}\left(\mathrm{X}_{1}, l\right)}{\frac{\partial \mathrm{C}}{\partial x}\left(\mathrm{X}_{1}, t\right)}=-h(t)
$$

De même on remplace (27) par

$$
\mathrm{D} \frac{\partial \mathrm{C}}{\partial x}\left(\mathrm{X}_{1}, t\right)=-\mathrm{D}^{\prime} \frac{\partial \mathrm{C}^{\prime}}{\partial x}\left(\mathrm{X}_{1}, t\right)
$$

(On est désormais ramené à un probleme à /ronlieres fixes: Lrouver les deux fonctions $(:)(x, t)$, définie pour ()$\leqslant x \leqslant \mathrm{X}_{1}$, el $\left(C^{\prime}(x, l)\right.$, délinie pour $\mathrm{X}_{1} \leqslant x \leqslant a$ et verriliant respectivement dans ces domaines les écpuations de firck (21) el (22), avec les conditions iniliales ch aux limiles (2:3), (24) (25) et, au point de raccord fixe $F_{1}$, les conditions approchées (34) et (35).

(ie probleme peut se traiter de diverses manieres, conduisant à des séries plus ou moins commodes selon les cas, pour loule valeur de la constante $\mathrm{X}_{1}$. Les calculs sont asse\% complicfués el nous nous bornerons à prendre pour $X_{1}$ la valeur particulière

$$
\mathrm{X}_{0}=\overline{\Lambda \mathrm{F}_{0}}
$$

Fo étant le point qui divise le segment $\Lambda \mathrm{A}^{\prime}$ dans le rapport $\frac{\sqrt{D}}{\sqrt{()^{\prime}}}$, c'est-à-dire

$$
\begin{aligned}
\mathrm{x}_{0} & =\frac{a \sqrt{\mathrm{D}}}{\sqrt{\mathrm{D}+\sqrt{\mathrm{D}^{\prime}}}}=\frac{a}{1+\sqrt{\delta}} \\
\left(\text { on pose ici } \frac{\mathrm{I})^{\prime}}{\mathrm{D}}\right. & =\dot{\delta})
\end{aligned}
$$

La suile montrera que celte valeur particuliere, outre les simplifications considérables qu'elle apporte au calcul a bien l'ordre de grandeur voulu pour assurer la validilé de l'approximation.

()n vérilicra sans peine que, $\gamma$ désignant toujours la solution réduite du probleme du inur, loutes les 
conditions posées sont remplies en prenant

$\mathrm{C}(x, l)=\mathrm{C}_{00} \gamma\left(\frac{x}{2 \mathrm{X}_{0}}, \frac{\mathrm{D} t}{4 \mathrm{X}_{0}^{2}}\right)$

$+\left(C_{0} \frac{\sqrt{ } \bar{D}^{\prime}-\sqrt{\mathrm{J}}}{\sqrt{\mathrm{D}^{\prime}}+\sqrt{\mathrm{D}}}-2 C_{0}^{\prime} \frac{\mathrm{D}^{\prime}}{\mathrm{D}+\sqrt{ } \mathrm{DD}^{\prime}}\right) \gamma\left(1-\frac{x}{2 \mathrm{X}_{0}}, \frac{\mathrm{D})}{4 \mathrm{X}_{0}{ }^{2}}\right)$

$C^{\prime}(x, t)=C_{0}^{\prime} \gamma\left(\frac{11-x}{2 X^{\prime}{ }_{0}}, \frac{\Gamma^{\prime} t}{4 X^{\prime}{ }_{0}^{2}}\right)$

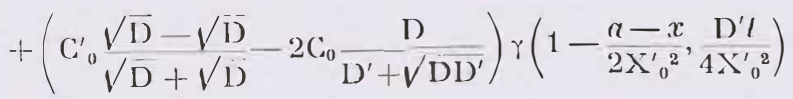

où on pose par symétrie

$$
\mathrm{X}_{0}^{\prime}=a-\mathrm{X}_{0}
$$

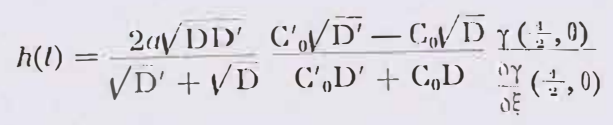

où

$$
0=\frac{\left.(\sqrt{\mathrm{D}})+\sqrt{\mathrm{D}^{\prime}}\right) \cdot \mathrm{l}}{4 a^{2}}
$$

On peut dresser une fois pour toule une lable de la lonction numéripue

$$
\psi(0)=2 \frac{\gamma\left(\frac{1}{2}, 0\right)}{\frac{\partial \gamma}{\partial \xi}\left(\frac{1}{1}, 0\right)}
$$

Les séries (32) ou (33) permetlent d'en obtenir (les expressions approchées

$$
\psi(0)=2 \sqrt{\pi 00} e^{\frac{1}{16 i i n}}\left(1-c+\frac{1}{4 \sqrt{0}}\right)
$$

valable pour

$$
0<\frac{1}{8}
$$

et

$$
\psi(0)=1-\frac{4}{\pi} e^{-\pi^{20}}+2 e^{-4 \pi \cdot 0}
$$

valable pour

$$
0>\frac{1}{8}
$$

$\Lambda$ partir de là on retrouve notamment la position asymptotique $\mathrm{I}_{p}$, du front, qui divise $\mathrm{A} \Lambda^{\prime}$ dans $\mathrm{le}$ rapport $\frac{\mathrm{C}_{n} \mathrm{D}}{\left.\mathrm{C}_{{ }_{\prime}^{\prime}} \mathrm{D}\right)^{\prime}}$ La loi de déplacement de front $\mathrm{F}$ au cours du lemps, donnéc par la formule (36), peut. dès lors se résumer sous la forme

נ. CH. PHYS. TOME $55(1958$ '

$$
\overline{F_{0} \mathbf{F}}=\overline{F_{0} \mathbf{F}_{p}}, \bigcup(0)
$$

Parmi les conclusions qu'on peut lirer de celle élude, la comnaissance de la loi selon laquelle F s'approche de la position asymptotique I" ${ }_{p}$ nous paraît particulièrement précieuse pour discuter et améliorer les déterminations quantilatives basées sur le pointe de $F_{p}$. Expérimentalement en effet, divers phénomènes parasiles interdisent de laisser la précipitation se poursuive trop longtemps : accumulation de précipité dans la zone finale balayéc par $F$, ce (qui perturl)e la diffusion, ou encore, dans certains montages, dessication du gel. On pourra désormais écourter l'expérience en évaluant la correction que cela implipue.

\section{Appareillage}

Les études expérimentales sont lailes à l’aide de deux montages fort simples.

10 Un monlage, permetlant des mesures rapides mais grossières, des pholographies à des lemps différents el une lacile observation microscopique, est réalisé à l'aide de plaques de verre photographicpue sur lespuelles on coule un gel d'épaisseur bien uniforme. Dans ce gel, on découpe des réservoirs rectangulaires allongés el parallèles destinés à recevoir les réactil's $R$ el $R^{\prime}$. Ceux-ci sont régulièrement renouvelés et l'ensemble est placé à l'abri de l'air dans une atmosphère salurée de vapeur d'eau pour éviter le dessèchement du gel, et maintenu à température constante. Une fois séchées, après diffusion, les placpues peuvent être passées au comparateur.

Ce type de montage permet de faire de bons dégrossissages en multipliant les systemes éludiés. Il permet aussi des vérifications approximatives d'expériences réaliscécs d'une laçon beaucoup plus précise.

2o Le montage précis comporte un lube de verre bien calibré clont la longueur peut varier entre 4 et $8 \mathrm{~cm}$. C.e tube horizontal, contenant le gel, est relié a deux réservoirs de grande capacilé renfermant $R$ et $R^{\prime}$. L'agitation de ces réactifs est assurée par un dégagement très lent de bulles d'air. L'ensemble est lixé sur la platine d'un comparateur dont la lunetle permet de viser les fronts du précipité dont la position peut être connue au $1 / 100$ de mum près. Ia précision n'est d'ailleurs pas limiléc par l'appareil de mesure, mais par la plus ou moins grande netleté du front.

\section{Résultats expérimentaux}

Ils vérifient souvent d'une façon convenable les prévisions théori(ues. Il est bon d'éviter, autant 
que possible, les systemes donnant des complications. Nous avons beaucoup utilise, comme réaclils, des sulliales solubles diflusant contre du chlorure. de baryum.

Nous constatons parfois, lor's de l'evolution de la diffusion, un dépassement du lront limite théorique $F_{p}$.

Parmi les causes d’erreur inévilables, nous signalons le gonflement du gel par les sels, qui provogue la formation de ménisques convexes aux extrémités du tube.

$\Lambda$ des lins de comparaison, nous voulons laire un monlage permetlant d'étudier la diflusion double dans l'eau pure enfermée dans un lube capillaire.

L'ensemble de nos résultats, dejjà important, justilie une publication ultérieure.

\section{Conclusion}

Nous avons construil un cadre idcéal, en partant d'hypothèses simples, dans un domaine oì les cas particuliers abondent (retards à la précipitation, solubilité du précipité dans l'un des réactils, protection de ce précipite par le gel, etc...). Il ne faut done pas s'altendre à ce que lous les systemes observés entrent exactement dans ce cadre.

Mais, précisément, l'un des gros intérêts de la question est de rechercher el d'in lerpréler pourchaque systeme étudié, les écarts entre les résultals des mesures el les lois que nous venons d'indiguer.

1)e Loule facon, nous pensons que notre travail doit contribuer à rendre plus quantitatives les méthodes d'analyse par diflusion.

\section{J3IBISOGIRAPIIIE}

(1) J. Striplan. - Wiener Silz., 1878, 77, 371.

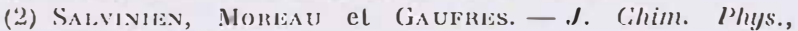
$1953,53,1$.

(3) Salvinilis el Gaubliss. - C. R, 1954, 238, 798.

\section{1) ISCUSSION}

1. Mamsun. - Save\%-vous si (les expériences semblalıles alux vótres ont élé faites avec des corps réagissant pour former un profluil soluble? On pourrail, par exemple, prendre un acicle et une base, ou un ion mílallicque et un agent complexant. si ce produit est triss stabje, la réaction se ferait dans un seul sens et l'on éliminerail la complication possible due à la formation de précipité.

J. Sulvinien. - On a effectivement fait des expériences (le ce genre, mais en (liffusion simple (méthode de l'inclicateur). Il serait intíressant de les faire en diffusion double, comme le suggere M. A(lamson. 